\title{
Higher order accurate solutions for flow in a cavity: experiences and lessons learned
}

\author{
Peter Eliasson $^{1}$, Marco Kupiainen ${ }^{2}$ and Jan Nordström ${ }^{2}$
}

\begin{abstract}
Experiences from using a higher order accurate finite difference multiblock solver to compute the time dependent flow over a cavity is summarized. The work has been carried out as part of a work in a European project called IDIHOM in a collaboration between the Swedish Defense Research Agency (FOI) and University of Linköping (LiU). The higher order code is based on Summation By Parts operators combined with the Simultaneous Approximation Term approach for boundary and interface conditions. The spatial accuracy of the code is verified by calculations over a cylinder by monitoring the decay of the errors of known wall quantities as the grid is refined. The focus is on the validation for a test case of transonic flow over a rectangular cavity with hybrid RANS/LES calculations. The results are compared to reference numerical results from a $2^{\text {nd }}$ order finite volume code as well as with experimental results with a good overall agreement between the results.
\end{abstract}

\section{Introduction}

The project IDIHOM (Industrialization of High-Order Methods - a Top-Down Approach) is a collaboration between 21 European partners from universities, research establishments and industries. The objective of the project is to industrialize the higher order methods and CFD codes to handle industrial type of applications. Within the scope of IDIHOM, FOI and LiU have jointly been further developing a higher order finite difference CFD code for multiblock structured grids. The current paper focuses on the validation of a calculation using a formally $3^{\text {rd }}$ order accurate

\footnotetext{
${ }^{1}$ Department of Aeronautics and Autonomous Systems, FOI, Swedish Defense Research Agency, SE-16490 Stockholm, Sweden, e-mail: peter.eliasson@foi.se

${ }^{2}$ Department of Mathematics, Computational Mathematics, University of Linköping, SE-581 83 Linköping, Sweden
} 
approach in space for calculations of the unsteady, turbulent transonic flow over a rectangular cavity with experimental comparisons.

The next section summarizes the computational approach including a short description of a tool that has been used to make reference calculations. Section 3 describes the verification for flow over a cylinder by a study of the decay of errors of known wall quantities as the grid is refined. The cavity flow calculations are then presented and in Section 4 we summarize and make concluding remarks.

\section{Computational tools}

The higher order finite difference solver, called Essense, is based on Summation By Parts (SBP) operators combined with the Simultaneous Approximation Term (SAT) approach with penalty terms that guarantee accuracy and stability [1]-[5]. The code is able to handle arbitrary order of spatial accuracy, but is currently limited to $5^{\text {th }}$ order. The code uses central difference operators for the approximation of the first derivative, $D U=P^{-1} Q U$, where $P$ is a block diagonal positive matrix containing the step size and where $Q$ is an almost block skew symmetric difference matrix. The Navier-Stokes equations are transformed to a curvilinear coordinate system where the differentiation is carried out separately in each direction. The transformation contains metric first derivatives which are differentiated with the same technique. Second differences, as in the viscous terms, are computed by applying the first difference operator twice. Since the difference operator is central, artificial dissipation is added to stabilize the computations [6]. No shock capturing has been applied for the computations described here.

Weak boundary conditions are applied on all boundaries including wall boundary conditions, far-field boundary conditions and interface conditions between blocks. A common feature for all boundary and interface conditions is that they are enforced through penalty terms multiplying the difference of the unknown quantity and the corresponding prescribed data. The data, the size of the penalties and the number of boundary conditions depend of the specific boundary condition. Explicit time stepping with a $4^{\text {th }}$ order accurate additive Runge-Kutta scheme is used to integrate the governing equations in time.

The parallel implementation utilizes domain decomposition for each block of the multiblock grid. Point-to-point communication is done by using so called halozones of half the width of the central SBP-operator. The communication across the different grids yields a possibly one-to-many and many-to-one communication pattern, which may have an adverse effect on load-balancing and scalability [7].

The higher order results for the cavity are compared to reference results from a formally $2^{\text {nd }}$ order accurate CFD solver, the Edge code, being an edge- and nodebased Navier-Stokes flow solver applicable for both structured and unstructured grids [8]-[10]. Edge is based on a finite volume formulation where a median dual grid forms the control volumes with the unknowns allocated in the centers. The 
governing equations are integrated with a multistage Runge-Kutta scheme to steady state and with acceleration by FAS agglomeration multigrid [11].

\section{Computed results}

\subsection{Verification for flow over a cylinder}

The computed results from the higher order code Essense have been verified for a number of test cases. Here we describe the verification for flow over a cylinder where the decay of errors of known wall quantities are studied as the grids are successively refined. The flow conditions are $M_{\infty}=0.3$ and $R e=50$ where the Reynolds number is based on the cylinder diameter. Five successively refined grids are used, the grids are of O-type structured grids where the coarsest grid contains $51 \times 51$ nodes. Two spatial operators denoted 42- and 84-operators are used where the first figure in the notation denotes the interior accuracy and the second the accuracy at the boundary. The two operators are formally $3^{\text {rd }}$ and $5^{\text {th }}$ order accurate, respectively.

Fig. 1 shows the decay of the errors of the wall velocity for a calculation with isothermal wall boundary conditions (left) and the errors of the normal wall temperature gradient using adiabatic boundary conditions (right). The errors follow more or less the expected design order; the decay of the errors is actually slightly higher than the design order. The results indicate the correct behavior and implementation of the numerical schemes.
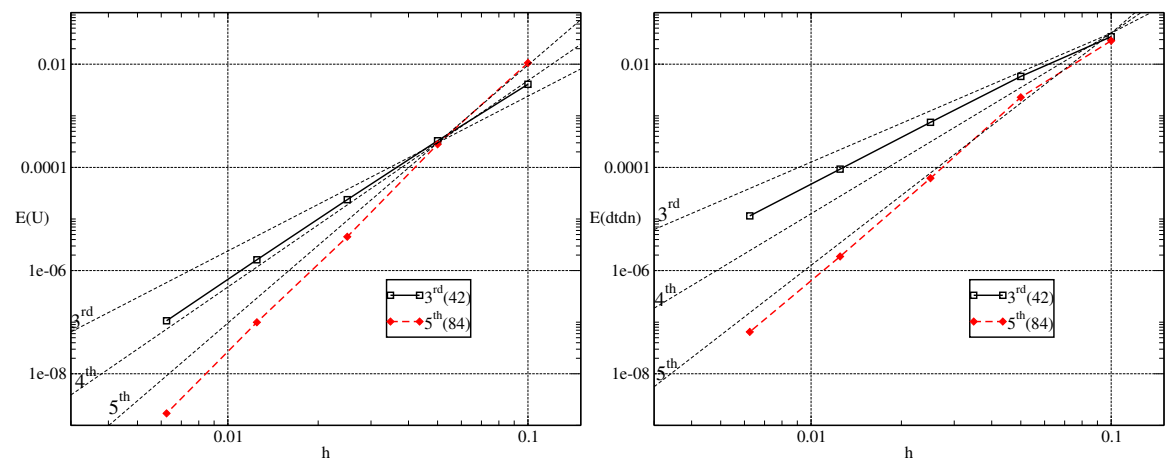

Fig. 1 Decay of errors of the velocity using isothermal wall boundary conditions (left); decay of temperature gradient using adiabatic wall boundary conditions (right). 


\subsection{Validation for flow over a cavity}

All partners within IDIHOM validated higher order results on different industrially relevant test cases. FOI and LiU chose a test case with transonic flow over a rectangular cavity, the test case is also denoted M219 in the literature [12]. The test case is suitable for Large Eddy Simulations (LES) or for hybrid RANS/LES calculations due to the turbulent fluctuations over the cavity. Experimental, time dependent data exist on the cavity walls and floor [12], computational results are available in many past references, e.g. [13],[14]. Several different cavity geometries exist; the one used here is the cavity with 5:1:1 length-to-depth-to-width relation. The geometry as well as the locations of the pressure probes are reproduced in Fig. 2.

The free stream values are $M_{\infty}=0.85$ and $R e=6.8 \times 10^{6}$ where the Reynolds number is based on the cavity length (20 inches). The cavity is experimentally measured on a device inside a wind tunnel. For the higher order calculations with Essense, calculations were carried out on a flat plate with the cavity embedded. A two block structured grid was created for these calculations where one block is located inside the cavity. To have a single boundary condition per block side, the block on top of the cavity block was split up resulting in 10 blocks all together. The computational grid is depicted in Fig. 3. The stretching of the grid near the boundaries is

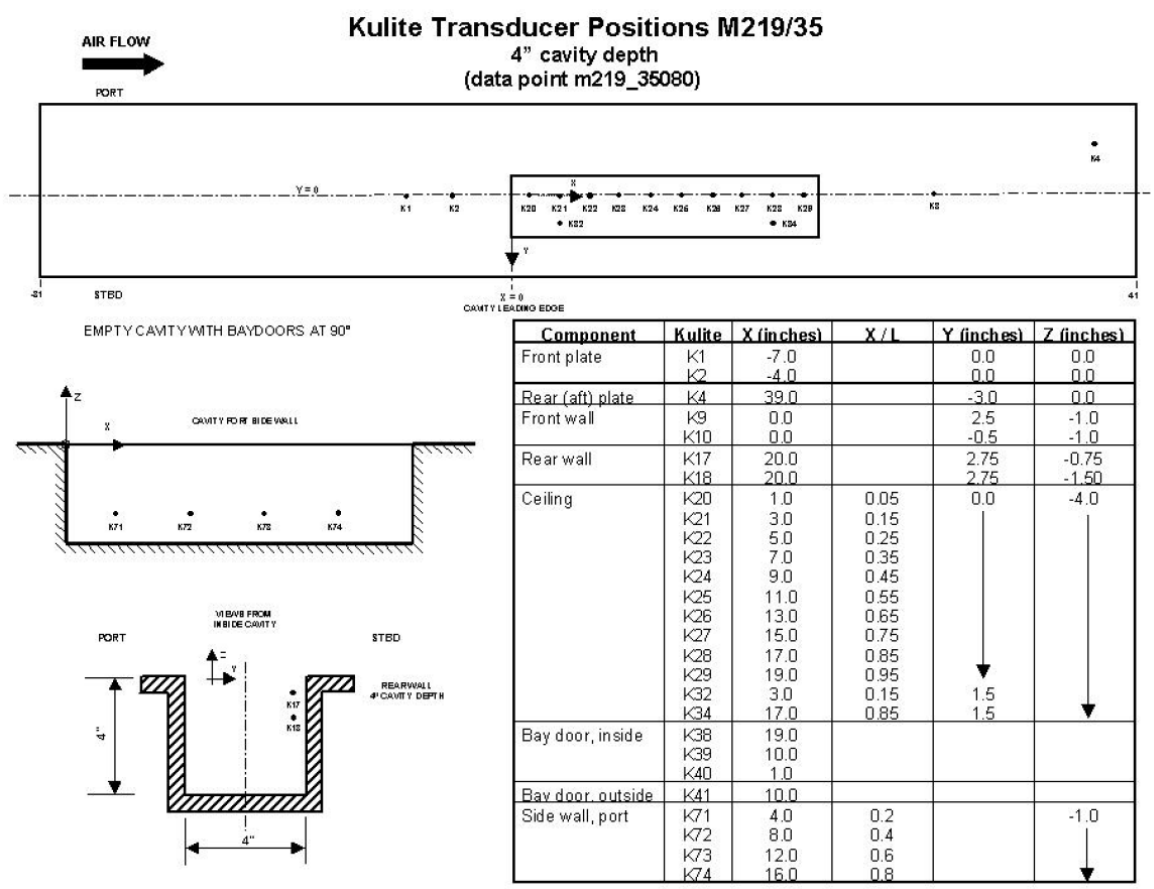

Fig. 2 Cavity geometry, experimental setup and location of the pressure probes recording unsteady pressure fluctuations [12]. 
relaxed in the interior to have a more uniform resolution which causes the grid to become curvilinear. For the reference calculations with Edge [15], the calculations were carried out on a hybrid unstructured grid that has been generated by EADS (European Aeronautic Defence and Space Company) and contains a grid over the cavity, the device on which the cavity is integrated and the entire test section of the wind tunnel. The main data of the two grids are displayed in Table 1. The grid for the higher order calculations has fewer grid points than that for the reference calculations with Edge.

Table 1 Details of the computational grids for the cavity

\begin{tabular}{|c|c|c|c|c|c|}
\hline Grid & Solver & \# vol. nodes & $\begin{array}{c}\text { \# boundary } \\
\text { nodes in cavity }\end{array}$ & $\begin{array}{c}\# \text { volume } \\
\text { nodes in cavity }\end{array}$ & $\begin{array}{l}\text { Near wall } \\
\text { distance }\end{array}$ \\
\hline Structured & Essense & $2.6 \times 10^{6}$ & $41 \times 10^{3}$ & $0.73 \times 10^{6}$ & $1.2 \times 10^{-5} \mathrm{~m}$ \\
\hline Unstructured & Edge & $6.2 \times 10^{6}$ & $77 \times 10^{3}$ & $2.0 \times 10^{6}$ & $4.0 \times 10^{-6} \mathrm{~m}$ \\
\hline
\end{tabular}

The computational grids are designed to carry out hybrid RANS/LES calculations with RANS in the near wall region and LES off wall in the cavity. Both calculations were initiated from poorly converged steady state calculations with local time steps. Only one higher order calculation was performed using a $3^{\text {rd }}$ order accurate calculation (42-operator), no model was used for modeling the turbulence. Adiabatic weak wall boundary conditions were used inside the cavity and on the plate. Far-field boundary conditions were used elsewhere. The higher order calculation use explicit time stepping and progress for about 60 through flows $\left(L / U_{\infty}\right.$ where $L$ is the cavity length and $U_{\infty}$ the free stream velocity), the solutions from last 40 through flows are used for the statistics.

The reference calculations use the $2^{\text {nd }}$ order implicit backward difference method and dual time stepping in each time step. An algebraic RANS/LES model is used to model the turbulence [15]. The calculations progress for about 120 through flows with statistics from the last 80 through flows. Some computational parameters are given in Table 2.
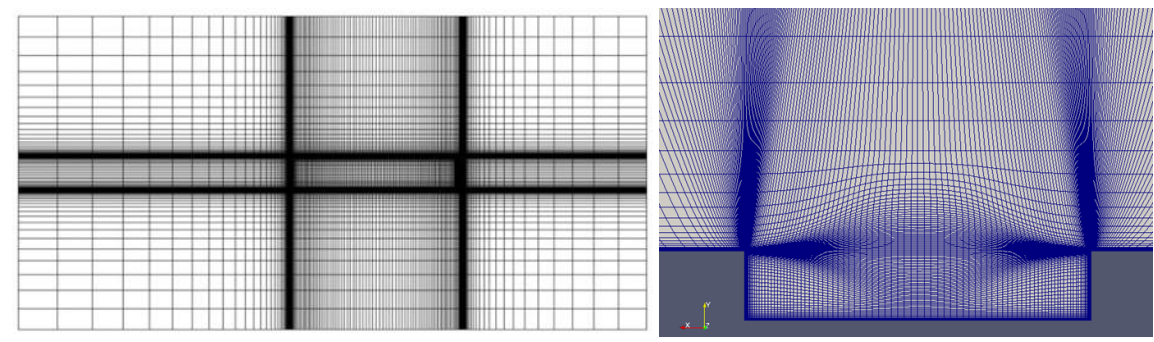

Fig. 3 Mesh pictures of the cavity test case used for Essense. 
Table 2 Sizes of time steps and number of inner iterations for the reference cavity calculations

\begin{tabular}{ccccc}
\hline Grid & Solver & $\Delta t$ & $\Delta t / T$ & $\mathrm{~N}$ inner iterations \\
\hline Structured & Essense & $1.0 \times 10^{-8}$ & $182 \times 10^{3}$ & 1 \\
Unstructured & Edge & $2.0 \times 10^{-5}$ & 91 & 32 \\
\hline
\end{tabular}

In Fig. 4 the sound pressure level (SPL) at two locations on the cavity floor are presented. The overall sound pressure level (OASPL) is displayed and compared to experimental values in Fig. 5, the OASPL is obtained by integrating SPL for all frequencies. The higher order results compare reasonably well to the experimental values of SPL. The main tonal peaks are captured. The higher order results have a tendency to have somewhat larger amplitudes of the oscillations compared to the reference results. This may be due to the lack of RANS/LES model for the higher order calculations or possibly a too short and coarse sampling interval. The computed OASPL from the higher order scheme and from the reference calculations with the unstructured grid agree well with the experimental values, an over prediction of OASPL is common.
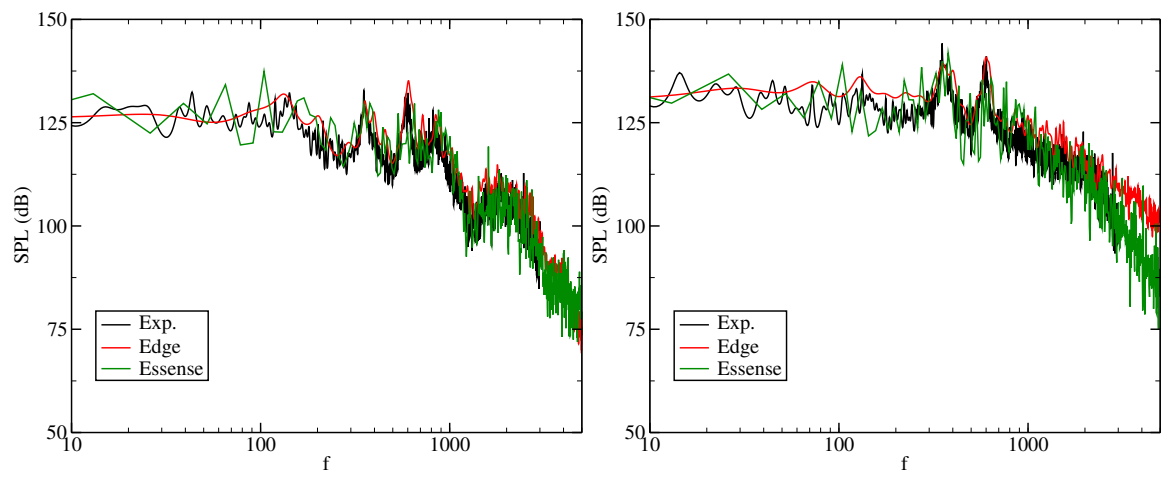

Fig. 4 Sound Pressure Level at cavity floor at kulits k21, $x / L=15 \%$ (left); $\mathrm{k} 25, x / L=55 \%$ (right).

Fig. 5 Overall Sound Pressure Level at cavity floor.

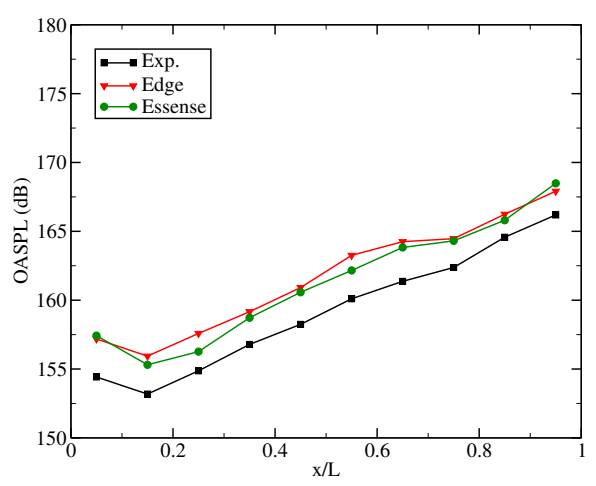


In an attempt to quantify the deviation from the experimental OASPL and to make a cross comparison between different results we defined the normalized deviation $D$ as

$$
D=\frac{\left\|O_{C F D}-O_{\exp }-\overline{\delta O}\right\|_{2}}{\bar{O}_{\exp }}=\frac{\sqrt{\frac{1}{N} \sum_{i=1}^{N}\left(O_{i, C F D}-O_{i, \exp }-\overline{\delta O}\right)^{2}}}{\bar{O}_{\exp }}
$$

where $\bar{O}_{\text {exp }}=159.2 d B, N=10$. The intention with the derived formula for the deviation is to define a measure that gives a zero value if the shape of OASPL is identical to the shape of the experimental OASPL. It allows for a shift in absolute level though. As it turns out the deviation obtained with the higher order scheme is the same as that obtained with Edge indicating that the two solutions follow experimental OASPL equally well.

\section{Summary and conclusions}

A higher order, provable stable, finite difference solver has been verified for a $2 \mathrm{D}$ cylinder flow case for which design order accuracy was obtained. The higher order solver has been applied to an industrially relevant case, the transonic flow over a $3 \mathrm{D}$ rectangular cavity at a high Reynolds number for which $3^{\text {rd }}$ order accurate time dependent solution were obtained. The quality of the solutions was good in terms of SPL and OASPL indicating that the higher order solution can be of use for industrial applications. The next phase in the development of Essense includes steady state convergence acceleration and implicit time integration.

Acknowledgements This work has been carried out within the EU project IDIHOM under contract No. FP7-AAT-2010-RTD-1-2657808.

\section{References}

1. Nordström, J., Eriksson, S., Eliasson, P.: Weak and Strong Wall Boundary Procedures and Convergence to Steady-State of the Navier-Stokes Equations. Journal of Computational Physics, 231, 4867-4884 (2012).

2. Berg, J., Nordström, J.: Superconvergent functional output for time-dependent problems using finite differences on summation-by-parts form. Journal of Computational Physics, 231, 68466860 (2012).

3. Berg, J., Nordström, J.: On the impact of boundary conditions on dual consistent finite difference discretizations. Journal of Computational Physics, 236, 41-55, (2013).

4. Carpenter, M., H., Nordström, J. and Gottlieb, D.: A Stable and Conservative Interface Treatment of Arbitrary Spatial Accuracy. Journal of Computational Physics, Vol 148 No. 2, 341365, (1999). 
5. Berg, J., Nordström, J.: Stable Robin Solid Wall Boundary Conditions for the Navier-Stokes Equations. Journal of Computational Physics, Vol 230, pp. 7519-7532, (2011).

6. Mattsson, K., Svärd, S. and Nordström, J.: Stable and Accurate Artificial Dissipation. Journal of Scientific Computing, Vol 21, No. 1, 57-79, (2004).

7. Rantakokko, J.: Partitioning strategies for structured multiblock grids, Parallel Computing, Vol. 26, pp. 1661-1680, (2000).

8. Eliasson, P., Weinerfelt, P. Recent Applications of the Flow Solver Edge. Proceedings to 7th Asian CFD Conference, Bangalore, India, (2007).

9. Eliasson, P., Nordström, J. and Weinerfelt, P.: Application of a line-implicit scheme on stretched unstructured grids. AIAA paper 2009-163, (2009).

10. Eliasson, P., Eriksson, S., Nordström, J.: The Influence of Weak and Strong Solid Wall Boundary Conditions on the Convergence to Steady-State of the Navier-Stokes Equations. AIAA Paper 2009-3551, (2009).

11. Brandt,A. and Livne, O. E.: Multigrid techniques, 1984 Guide with Applications to Fluid Dynamics, in Applied Mathematics, SIAM, 67, ISBN 978-1611970746, (1984).

12. Chen, X., Sandham, N., D., Zhang, X.: Cavity Flow Noise Predictions, Report No. AFM07/05, School of Engineering Sciences, University of Southampton (2007).

13. Ashworth, R.M.: Prediction of acoustic resonance phenomena for weapon bays using detached eddy simulation, Aeronautical Journal, Vol. 109, No. 1102, 631-638, (2005).

14. Peng, S.-H. Simulation of Flow Past a Rectangular Open Cavity using DES and unsteady RANS, AIAA Paper 2006-2827 (2006).

15. Peng, S.-H.: Hybrid RANS-LES modelling based on zero- and one-equation models for turbulent flow simulation. In Proceedings of 4th Int. Symp. Turb. and Shear flow Phenomena, Vol. 3, pages 1159-1164 (2005). 